\title{
ARTICLE
}

Genetics and Genomics

\section{Negative plasma Epstein-Barr virus DNA nasopharyngeal carcinoma in an endemic region and its influence on liquid biopsy screening programmes}

\author{
John Malcolm Nicholls ${ }^{1}$, Victor Ho-Fun Lee ${ }^{2,3}{ }^{2}$, Sik-Kwan Chan ${ }^{2}$, Ka-Chun Tsang ${ }^{2}$, Cheuk-Wai Choi ${ }^{2}$, Dora Lai-Wan Kwong ${ }^{2,3}$, \\ Ka-On Lam ${ }^{2,3}$, Sum-Yin Chan ${ }^{2}$, Chi-Chung Tong ${ }^{2}$, Tsz-Him So ${ }^{2}$, To-Wai Leung ${ }^{2,3}$, Mai-Yee Luk ${ }^{2}$, Pek-Lan Khong ${ }^{4}{ }^{4}$ and \\ Anne Wing-Mui Lee Le,3 $^{2,3}$
}

BACKGROUND: Epstein-Barr virus (EBV)-associated nasopharyngeal carcinoma (NPC) in endemic regions may have undetectable plasma EBV DNA.

METHODS: We prospectively recruited 518 patients with non-metastatic NPC and measured their pre-treatment plasma EBV DNA. The stage distribution and prognosis between pre-treatment plasma EBV DNA-negative (0-20 copies/ml) and EBV DNA-positive (>20 copies $/ \mathrm{ml}$ ) patients following radical treatment were compared.

RESULTS: Seventy-eight patients (15.1\%) were plasma EBV DNA-negative, and 62 in this subset (12.0\%) had 0 copy/ml. Only $23 / 78$ (29.5\%) plasma EBV DNA-negative patients with advanced NPC (stage III-IVA) had strong EBV encoded RNA (EBER) positivity (score 3 ) in their tumours compared to $342 / 440$ (77.7\%) EBV DNA-positive patients of the same stages $(p<0.001)$. Though EBV DNAnegative patients had more early-stage disease $(p<0.001)$ and smaller volumes of the primary tumour and the positive neck nodes $(p<0.001)$, they had similar 5-year overall survival and cancer-specific survival to those EBV DNA-positive counterparts by stage. Similar results were also seen when plasma EBV DNA cut-off was set at 0 copy/ml.

CONCLUSIONS: Patients with low-volume NPC may not be identified by plasma/serum tumour markers and caution should be taken in its utility as a screening tool for NPC even in endemic regions.

CLINICAL TRIAL REGISTRATION: Clinicaltrials.gov Identifier: NCT02476669.

British Journal of Cancer (2019) 121:690-698; https://doi.org/10.1038/s41416-019-0575-6

\section{INTRODUCTION}

Nasopharyngeal carcinoma (NPC) is endemic in southern China including Hong Kong. ${ }^{1}$ Over the past 20 years, plasma Epstein-Barr Virus (EBV) deoxyribonucleic acid (DNA) has been advocated for the diagnosis of NPC. The rationale for this methodology has been based on the concept that since EBV has been associated with NPC, the circulating viral or tumour associated DNA (ctDNA) may be detected in the blood as a measure of tumour presence or disease burden. However, in NPC there is a sensitivity between 53 and $96 \%,{ }^{2}$ and this wide variation has been attributed to three main factors - the analytical method of detection, disease stage and the World Health Organization (WHO) histology of the disease. There has been an attempt to develop standardised protocols of analysing plasma EBV DNA, ${ }^{3,4}$ but meta-analyses of plasma EBV DNA in the diagnosis of NPC have not reported the proportion of plasma EBV DNA-positive patients for each WHO type, and only four studies looked at the clinical stage. ${ }^{5-7}$ Squamous cell carcinomas have typically been separated by WHO from non-keratinising carcinoma, as previous studies suggested that the former was usually negative for EBV by insitu hybridisation (ISH), while the other subtypes were positive. In Hong Kong, however, tumours with squamous differentiation can be EBV positive, ${ }^{8}$ and this has also been shown in NPCs from Malaysia. 9

Hong Kong is one of the first regions in the world discovering the association of plasma EBV DNA with NPC, and devised the first quantitative assay for accurate and reliable measurement. ${ }^{10}$ Over the years, the lowest detection limit as a representation of improved sensitivity has decreased from 60 copies $/ \mathrm{ml}$ to 20 copies $/ \mathrm{ml}^{11,12}$ and in a large NPC screening programme this low detection limit has been advocated as an initial screening tool. ${ }^{12}$ In this publication, we focused our attention on the incidence of plasma EBV DNA-negative ( $\leq 20$ copies $/ \mathrm{ml}$ ) NPC patients, their clinico-pathological characteristics and survival outcomes, in an attempt to determine whether there are unique features concerning these patients which may account for the absence of EBV DNA in their plasma. Furthermore, since there have been recent publications on the value of liquid biopsy in screening

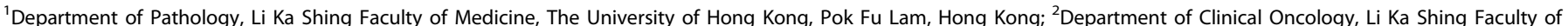

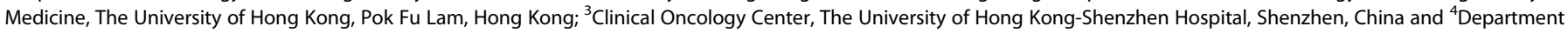
of Diagnostic Radiology, Li Ka Shing Faculty of Medicine, The University of Hong Kong, Pok Fu Lam, Hong Kong

Correspondence: Victor Ho-Fun Lee (vhflee@hku.hk)

These authors contributed equally: John Malcolm Nicholls, Victor Ho-Fun Lee

Received: 7 April 2019 Revised: 19 August 2019 Accepted: 27 August 2019

Published online: 17 September 2019 
programmes, ${ }^{13}$ we sought to determine whether the same concerns on the usefulness of these screening programmes could be applied to NPC.

\section{METHODS}

Study population

The study cohort comprising 518 consecutively and prospectively recruited patients with previously untreated non-metastatic NPC (i.e. stage I-IVA) enrolled in another study which investigated the role of plasma EBV DNA in proposing a new staging system (ClinicalTrials.gov NCT02476669). ${ }^{14}$ All biopsy-confirmed patients had complete staging investigations, including positron-emission tomography with integrated contrast-enhanced computed tomography (PET-CT) scan, magnetic resonance imaging (MRI), serum haematology, biochemistry and lactate dehydrogenase, serology for EBV immunoglobulin A ( $\lg A)$ for viral capsid antigen (VCA), and plasma EBV DNA to investigate the prognostic role of plasma EBV DNA taken at several time points before and after radical treatment (described further in Supplementary Material). ${ }^{15}$ The protocol and assay for plasma EBV DNA extraction and quantification for all patients in this study was the same as the one devised by Lo et al. ${ }^{10,12}$ In brief, all patient blood samples contained in EDTA tubes were immediately stored in a $4{ }^{\circ} \mathrm{C}$ refrigerator after blood taking from patients and they were processed for subsequent EBV DNA extraction within $4 \mathrm{~h}$ of blood taking in the single laboratory of our institution (further details on EBV DNA quantification and validation methods were described in Supplementary Material). A total of about $400-800 \mu \mathrm{l}$ of plasma samples were used for DNA extraction by a QIAamp Blood Kit (Qiagen, Hilden, Germany). The exact amount of plasma was determined for calculation of EBV DNA genome copies. Circulating EBV DNA concentrations were measured using a real-time quantitative polymerase chain reaction (PCR) system with $A B I$ Prism $^{\circledR} 7000$ Sequence Detection System (Applied Biosystems, USA) that amplified a DNA segment in the BamHI-W fragment region of the EBV genome. All samples were repeated twice on the same day by the same assay for accurate quantification and the results showed that the discrepancy was less than $2 \%$ for all repeated samples. The results were expressed as EBV DNA genome copies per $\mathrm{ml}$ with accuracy to the nearest 0.1 copies/ $\mathrm{ml}^{14}$ All pre-treatment investigations were performed within 14 days of the pathological diagnosis of NPC. All patients, within 14 days of these investigations, then received radical intensitymodulated radiation therapy (IMRT) with or without concurrent chemotherapy and adjunct (induction or adjuvant) chemotherapy based on the stage of the disease according to the 7th edition of American Joint Committee on Cancer (AJCC)/Union for International Cancer Control (UICC) TNM staging system. The details of treatment and follow-up surveillance were previously described. ${ }^{14,15}$ The TNM stage of each patient's disease was restaged according to 8th edition of AJCC/UICC TNM staging system for subsequent analysis in this study. ${ }^{14}$

As there has been no consensus on what determines the lowest limit of plasma EBV DNA detection, we selected and analysed patients who had 0-20 copies of EBV DNA per $\mathrm{ml}$ in the plasma as this has been regarded as the lowest limit of detection threshold in a recently reported screening study, with plasma EBV DNA $\leq 20$ copies $/ \mathrm{ml}$ (i.e. 0-20 copies $/ \mathrm{ml}$ ) designated as plasma EBVnegative. $^{12}$ In this publication, plasma EBV DNA-negative NPC and EBV DNA-positive NPC were defined as those diagnosed in patients who had pre-treatment $\leq 20$ copies $/ \mathrm{ml}$ and $>20$ copies $/ \mathrm{ml}$ respectively.

Histology and ISH Epstein-Barr virus-encoded RNA (EBER) analysis All NPCs in this study were classified according to WHO criteria into keratinising squamous cell carcinoma, non-keratinising differentiated carcinoma and non-keratinising undifferentiated carcinoma. The formalin-fixed paraffin-embedded (FFPE) tumour slides were subjected to ISH using the commercially available Inform EBER ISH probe (Ventana). They were scored based on the percentage of tumour cells positively stained with EBER as follows: 0 (no tumour cells positively stained); 1 (1-10\% tumour cells positively stained); 2 (11-50\% tumour cells positively stained), and 3 ( $>50 \%$ tumour cells positively stained) blindly by 2 independent pathologist and oncologist (JN and VL), suggested by Bar-Sela et al. ${ }^{16}$ An example of microscopic appearance of each EBER intensity by ISH $(0-3)$ in 4 patients of this study cohort was shown (Fig. S1). An excellent agreement (Cohen's Kappa 0.83) was observed and any discrepancy in scoring was resolved by consensus. An additional 85 cases of histologically confirmed NPC from 2013 to 2017 with plasma EBV DNA titres $\geq 30$ copies $/ \mathrm{ml}$ were used as positive controls.

Tumour volume analysis

The pre-treatment gross tumour volumes (GTV) of the primary nasopharyngeal tumour (GTV_P) and the radiologically positive neck nodes (GTV_N) of the contrast-enhanced PET-CT images of 3 $\mathrm{mm}$ slice thickness with reference to the co-registered MRI images were contoured manually by the treating clinical oncologists in Eclipse Treatment Planning System version 13.0 (Palo Alto, USA), which was also used for subsequent IMRT optimisation, as previously described. ${ }^{14,15}$ The resulting sum of the areas of the GTV_P and GTV_N was calculated by this treatment planning system to generate the respective volumes.

\section{Statistical analysis}

The pre-specified survival endpoints in this study included progression-free survival (PFS), overall survival (OS) and cancerspecific survival (CSS) as we defined previously. ${ }^{14,15}$ Kaplan-Meier methods were performed for these survival outcomes. Log-rank tests were employed to compare survival differences between plasma EBV DNA-negative and EBV DNA-positive patients. Association between EBER scores and T-, N- and overall stage of NPC as well as pre-treatment plasma EBV DNA subgroups (0-20 copies $/ \mathrm{ml}$ vs $>20$ copies $/ \mathrm{ml}$ and 0 copy $/ \mathrm{ml}$ vs $>0$ copy $/ \mathrm{ml}$ ) was performed by Chi-square tests. Statistical significance was defined as $p<0.05$ (two-sided). All statistical analyses were performed by Statistical Package for Social Sciences (SPSS) version 24.

\section{RESULTS}

From October 2010 to May 2016, 518 patients were prospectively recruited with their dispositions shown (Fig. 1, Table 1). Seventyeight (15.1\%) patients were classified as pre-treatment plasma EBV DNA-negative (i.e. $\leq 20$ copies $/ \mathrm{ml}$ ). Sixty-two $(79.5 \%)$ patients had 0 copy $/ \mathrm{ml}$ and $16(20.5 \%)$ had $1-20$ copies/ml of plasma EBV DNA before treatment. Pre-treatment plasma EBV DNA correlated very well with GTV_P, GTV_N, GTV_T + N and serum lactate dehydrogenase (all $p<0.01$ ) (Fig. S2). However, no correlation was identified between pre-treatment plasma EBV DNA and GTV_P $(p=0.936), \operatorname{GTV} \_\mathrm{N}(p=0.900)$ and GTV_T $+\mathrm{N}(p=0.910)$ in plasma EBV DNA-negative patients. Plasma EBV DNA-negative patients tended to have an earlier stage of their NPC, as well as smaller volumes of the primary tumour in the nasopharynx and the positive neck nodes. The overall stage distribution of these 78 plasma EBV DNA-negative patients was: stage I in $18(23.1 \%)$; stage II in $17(21.8 \%)$; stage III in $34(43.6 \%)$ and stage IVA in 9 (11.5\%). No plasma EBV DNA-negative patients had stage IVB metastatic disease at the time of initial diagnosis. The T- and Nclassification of the plasma EBV DNA-negative patients were shown (Table S1).

After a median follow-up of 5.2 years (range 1.2-6.4 years), the 5 -year PFS, OS and CSS of the whole population were $72.9 \%$, $79.8 \%$ and $86.1 \%$, respectively. Except for PFS $(92.7 \%$ vs $70.0 \%$, $95 \% \mathrm{Cl}=83.3-97.2 \%$ vs $73.1-82.8 \%, p=0.023)$, OS $(91.9 \%$ vs 


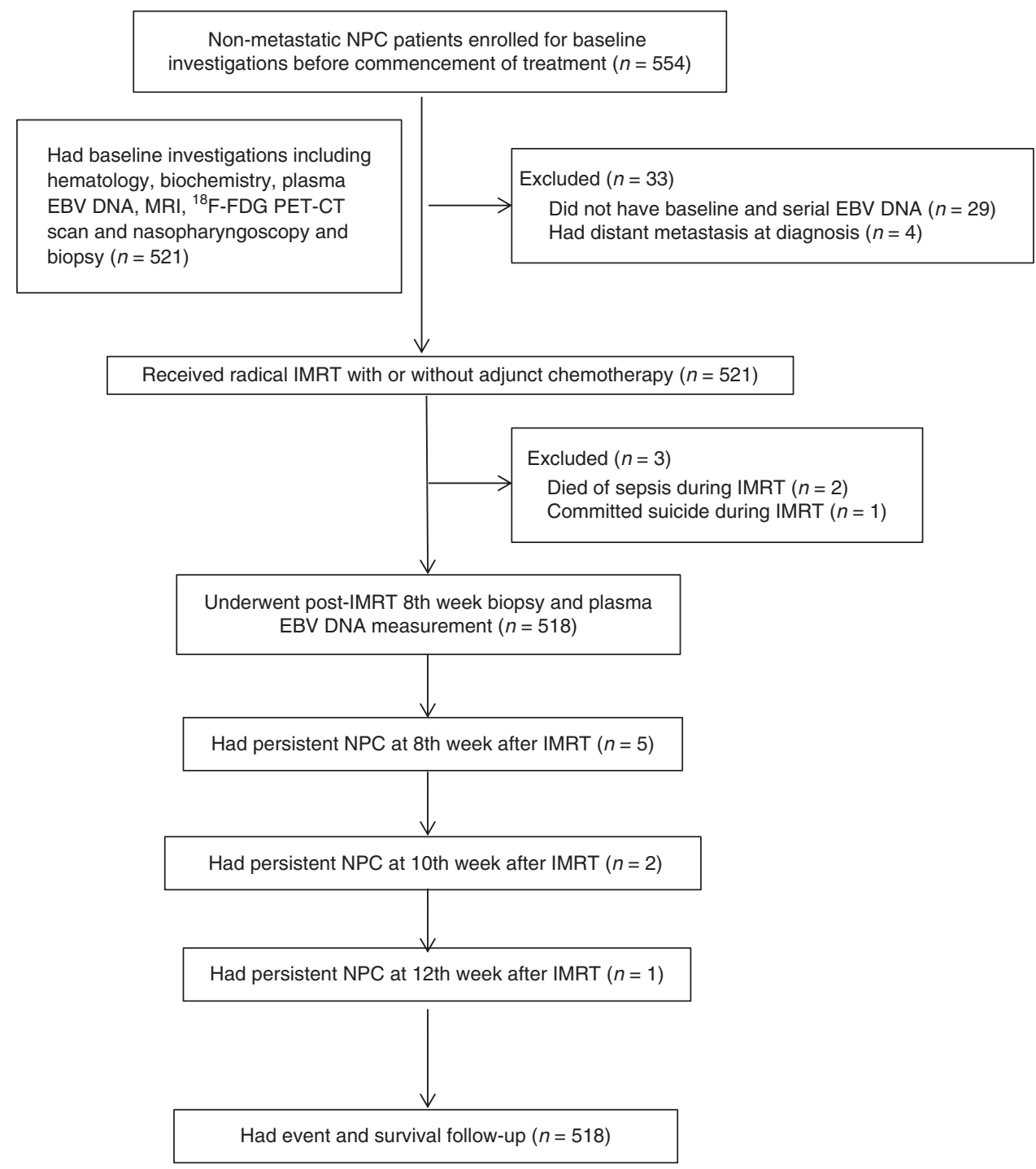

Fig. 1 Study flowchart

$78.1 \%, 95 \% \mathrm{Cl}=81.1-97.1 \%$ vs $73.1-82.8 \%, p=0.242)$ and CSS (96.2\% vs $84.6 \%, 95 \% \mathrm{Cl}=85.2-99.0 \%$ vs $80.0-88.3 \%, p=0.293)$ of the plasma EBV DNA-negative patients were not statistically better than their EBV DNA-positive counterparts (Table S2). When compared by stage, their survival outcomes (PFS, OS and CSS) were also not different from the plasma EBV DNA-positive patients (Figs. 2-4).

Since there were 62 and 16 patients who had 0 copies $/ \mathrm{ml}$ and 1-20 copies/ml of pre-treatment plasma EBV DNA, respectively, we also compared the stage distribution, tumour and nodal volumes and survival endpoints with those who had pretreatment plasma EBV DNA >20 copy/ml (Tables S3-S6). Again, patients with pre-treatment plasma EBV DNA 0 copy/ml had more early-stage disease, smaller tumour and nodal volumes but similar survival outcomes (except PFS) as compared to those who had pre-treatment plasma EBV DNA >0 copy/ml (Figs. S3-S5 and Table S7).

Association of EBER with plasma EBV DNA and overall stage of disease

The association of EBER by ISH with plasma EBV DNA and NPC T-, $\mathrm{N}$ - and overall stage was shown (Tables S8-S13). Though statistical significances on the association between EBER scores and overall stage were identified in the whole study population and plasma
EBV DNA-positive patients, they were not detected in plasma EBV DNA-negative patients. Intriguingly, only 23 out of $78(29.5 \%)$ of advanced NPC (stage III-IVA) patients who were plasma EBV DNA-negative had diffuse strong EBER positivity (score 3 ) in their tumours, as compared to 342 of $440(77.7 \%)$ plasma EBV DNA-positive patients of the same stages $(p<0.001)$. Plasma EBV DNA-negative patients were also associated with a lower EBER intensity in their tumours $(p<0.001)$ (Table S11). The results were also similar when 0 copy $/ \mathrm{ml}$ was set as the cut-off: 41 of $62(66.1 \%)$ had EBER score 3 in patients with plasma EBV DNA 0 copy $/ \mathrm{ml}$ compared to 412 of $456(90.4 \%)$ in patients with plasma EBV DNA $>0$ copy/ml $(p<0.001)$ (Tables S11-S13).

\section{DISCUSSION}

In view of the close association between NPC and EBV, antibodies to EBV have been used for NPC screening and treatment monitoring, and to differentiate NPCs from other head and neck cancers. ${ }^{17,18}$ Immunohistochemistry and ISH studies conclusively demonstrated that the EBV genome was incorporated into the tumour cells, ${ }^{19-21}$ resulting in EBV being classified as an oncogenic virus. ${ }^{22}$

In the late 1990's several studies showed that cancer-derived cells or DNA could be detected in the blood of cancer 
Table 1. Patient characteristics at baseline based on 8th edition of AJCC/UICC staging classification stratified by pre-treatment plasma EBV DNA (0-20 copies $/ \mathrm{ml}$ vs $>20$ copies $/ \mathrm{ml}$ )

\begin{tabular}{|c|c|c|c|c|}
\hline \multirow[t]{2}{*}{ Characteristic } & \multicolumn{3}{|l|}{ Patients, No. (\%) } & \multirow[t]{2}{*}{$p$} \\
\hline & Total $(n=518)$ & $0-20$ copies $/ \mathrm{ml}(n=78)$ & $>20$ copies $/ \mathrm{ml}(n=440)$ & \\
\hline Median age in years (range) & $53(16-90)$ & $54(16-86)$ & $53(16-90)$ & 0.983 \\
\hline Male/female & $385(74.3) / 133(25.7)$ & $61(78.2) / 17(21.8)$ & $324(73.6) / 116$ (26.4) & 0.395 \\
\hline Histology & & & & 0.405 \\
\hline Non-keratinising undifferentiated carcinoma & $508(98.1)$ & $78(100)$ & $430(97.8)$ & \\
\hline ECOG performance status & & & & 0.507 \\
\hline 0 & $80(15.4)$ & $14(17.9)$ & $66(15)$ & \\
\hline 1 & $438(84.6)$ & $64(82.1)$ & $374(85)$ & \\
\hline T-classification & & & & $<0.001$ \\
\hline N-classification & & & & $<0.001$ \\
\hline No & $60(11.6)$ & $25(32.1)$ & $35(8.0)$ & \\
\hline N1 & $127(24.5)$ & $21(26.9)$ & $106(24.1)$ & \\
\hline N2 & $201(38.8)$ & $27(34.6)$ & $174(39.5)$ & \\
\hline N3 & $130(25.1)$ & $5(6.4)$ & $125(28.4)$ & \\
\hline Overall stage & & & & $<0.001$ \\
\hline 1 & $30(5.8)$ & $18(23.1)$ & $12(2.7)$ & \\
\hline II & $74(14.3)$ & $17(21.8)$ & $57(13.0)$ & \\
\hline III & $234(45.2)$ & 34 (43.6) & $200(45.5)$ & \\
\hline IVA & $180(34.7)$ & $9(11.5)$ & $171(38.8)$ & \\
\hline Laterality of primary tumour & & & & 0.796 \\
\hline Stage IVA & $2012.5(0-1143750)$ & $0(0-14)$ & $2203(38-1143750)$ & 0.584 \\
\hline $\begin{array}{l}\text { Median pretreatment serum lactate dehydrogenase in } \\
\text { international units/litre (range) }\end{array}$ & $196(109-688)$ & $180.5(125-310)$ & $196(109-688)$ & 0.011 \\
\hline Stage I & $179.5(121-310)$ & $178.5(132-310)$ & $190.5(121-260)$ & 0.655 \\
\hline Stage II & $185.5(140-275)$ & $174(143-275)$ & $188(140-256)$ & 0.235 \\
\hline Stage III & $197.5(109-521)$ & $191(137-306)$ & $198(109-521)$ & 0.593 \\
\hline Stage IVA & $200(125-688)$ & $182(125-254)$ & $204(130-688)$ & 0.174 \\
\hline $\begin{array}{l}\text { Median Gross tumour volume of the primary tumour (GTV_P) } \\
\left(\mathrm{cm}^{3}\right)(\text { range) }\end{array}$ & $8.7(0-136)$ & $4.4(0-66.9)$ & $10.1(0-136)$ & 0.004 \\
\hline $\begin{array}{l}\text { Median Gross tumour volume of the positive neck nodes (GTV_N) } \\
\left(\mathrm{cm}^{3}\right) \text { (range) }\end{array}$ & $17.1(0-191.3)$ & $9.45(0.4-191.3)$ & $18.55(0-168.2)$ & $<0.001$ \\
\hline $\begin{array}{l}\text { Median Gross tumour volume of the primary tumour and the } \\
\text { positive neck nodes }\left(G T V \_P+N\right)\left(\mathrm{cm}^{3}\right)(\text { range) }\end{array}$ & $31.4(0.9-229)$ & $16.25(2.3-199.9)$ & $34.9(0.9-229)$ & $<0.001$ \\
\hline Radical IMRT only & $71(13.7)$ & $26(33.3)$ & $45(10.2)$ & $<0.001$ \\
\hline Concurrent chemoradiation & $91(17.6)$ & 14 (17.9) & 77 (17.5) & 0.194 \\
\hline Induction chemotherapy then concurrent chemoradiation & $165(31.9)$ & $12(15.4)$ & $153(34.8)$ & 0.022 \\
\hline Concurrent chemoradiation then adjuvant chemotherapy & $191(36.9)$ & 27 (34.6) & $164(37.3)$ & 0.199 \\
\hline
\end{tabular}




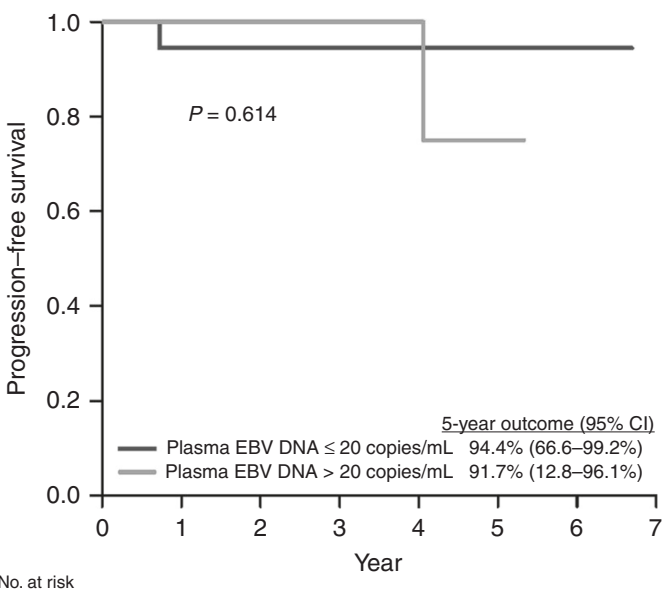

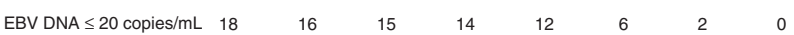

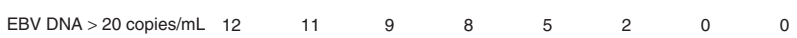

C

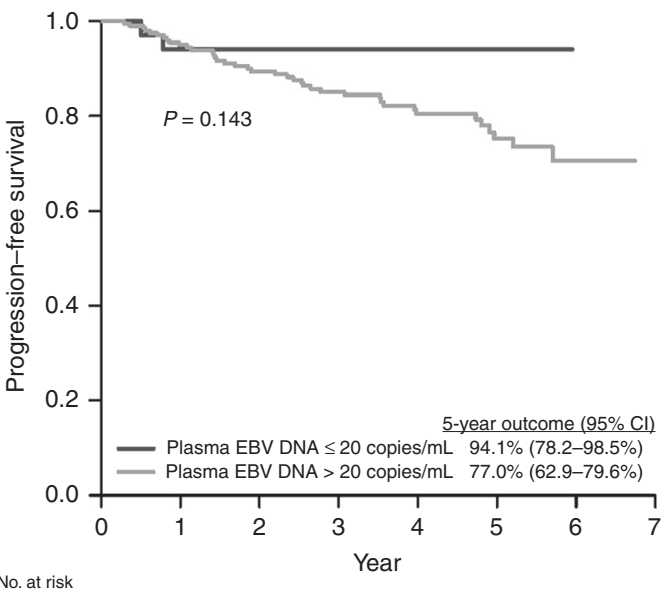

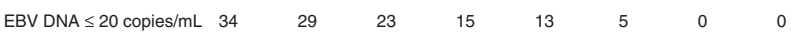

EBV DNA > 20 copies $/ \mathrm{mL} 200 \quad 182 \quad 155 \quad 130 \quad 92 \quad 51 \quad 14 \quad 140$ b

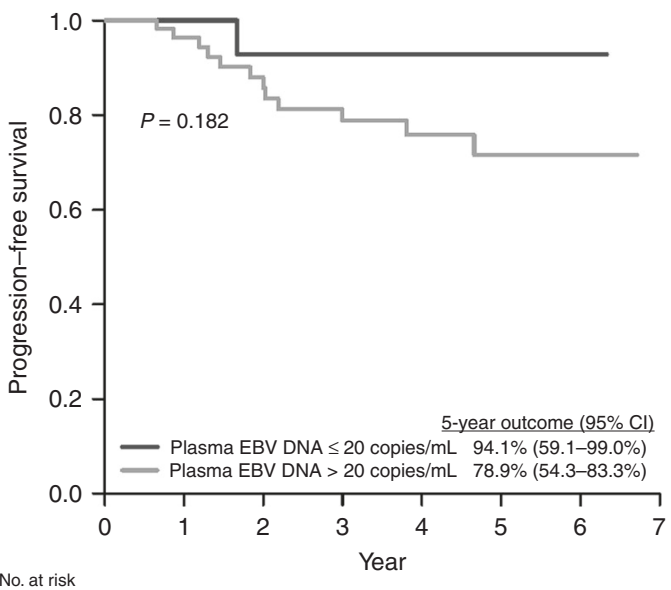

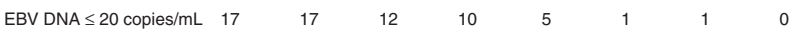

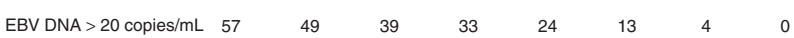

d

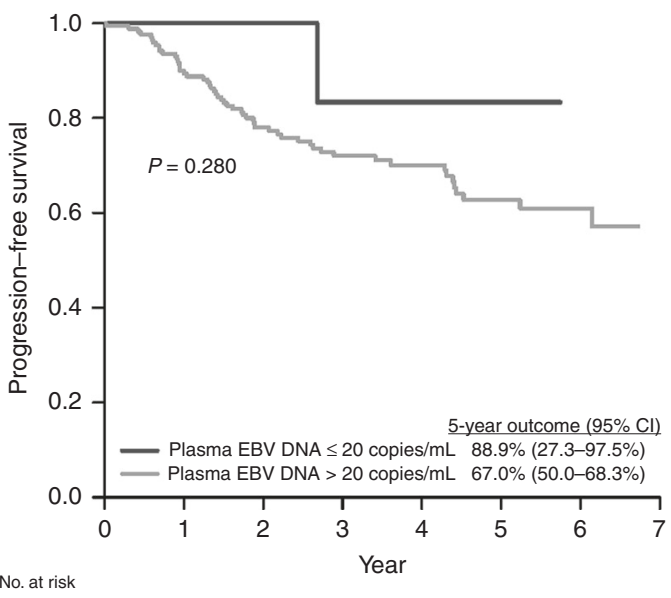

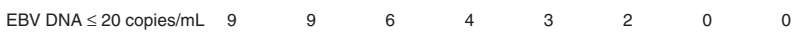

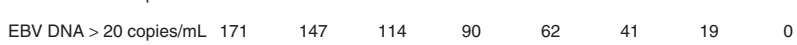

Fig. 2 Progression-free survival of NPC patients in the study population stratified by pre-treatment plasma EBV DNA (0-20 copies/ml vs $>20$ copies/ml). a Stage I. b Stage II. c Stage III. d Stage IVA

patients, ${ }^{23,24}$ and this was applied to NPC first by Mutirangura et al and later by Lo et al. ${ }^{25,26}$ Using the BamH1-W and EBNA1 targets to amplify, 55 of 57 patients had plasma EBV DNA detected compared to 3 of 43 normal individuals, giving a detection rate of $96 \%$. The false positive rate was $8 \%$. A recent review, however, showed that the sensitivity has ranged from $53-96 \%{ }^{2}$

The superiority of plasma EBV DNA over serum IgA VCA was later shown in 2004 by Shao et al, who also demonstrated that plasma EBV DNA correlated with the TNM stage, ${ }^{27}$ though IgA VCA was better at detecting stage I compared to stage IV disease. Plasma EBV DNA also appeared to be linearly related to T-stage which was suggested to be a measure of tumour burden. Leung et al in the same year also investigated the accuracy of combination of plasma EBV DNA and IgA VCA in detecting NPC. ${ }^{11}$ Using a detection limit of 60 copies/ml, EBV DNA was detected in 132/139 patients, with an increased sensitivity seen in later stages (87/89) than early stages $(45 / 50)$. The wide range of sensitivity previously reported may be due to many factors such as extraction technique, amplification technique and whether the BamH1-W region (which has more repeats for detection, but random in the number of repeats), or single-copy genes (e.g. EBNA1) are used as targets. ${ }^{28}$ Other potential factors that may contribute to assay include failed extraction, inhibition, patient age and the ambient temperature when blood is taken from patients. ${ }^{29,30}$ A previous report has shown low copy number of EBV in $3-7 \%$ of the general population when the BamH1-W target was used, and $2.03 \%$ when the EBNA1 target was used (1-512 copies $/ \mathrm{ml}$ ), so at these low numbers there is an overlap between normal subjects and patients with stage I NPC. ${ }^{31}$

In the screening programme performed by Chan et al., $9 \%$ of normal individuals had detectable plasma EBV DNA in the winter season and $5 \%$ in the summer, in which 20 copies $/ \mathrm{ml}$ was the lowest limit of detection. ${ }^{30}$ On the other hand, when 0 copies $/ \mathrm{ml}$ was used as a cut-off, plasma EBV DNA identified 17/24 NPC patients in a non-endemic (but high-risk population) area. ${ }^{32}$

The challenge of a screening tool for cancer is the balance between sensitivity and specificity. Recent publications have addressed the community acceptance of a false positive rate in cancer screening programs, ${ }^{33,34}$ where it has been recognised that there is a negative psychological outcome in patients with false positive screening in breast and lung cancer. ${ }^{35,36}$ Yip and colleagues commented that the lowest sensitivity of plasma EBV DNA for NPC is in the stage I patients-the major targets of screening programmes, but this review has been hampered by the very small number of patients included with this stage (ranging from 1 to 14 patients in each respective study) and the wide range 
a

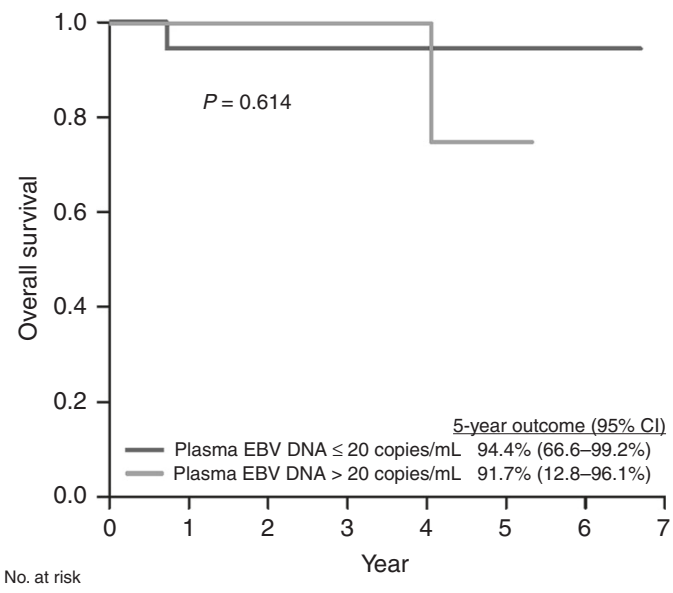

$\begin{array}{lllllllll}\text { EBV DNA } \leq 20 \text { copies } / \mathrm{mL} & 18 & 16 & 15 & 14 & 12 & 6 & 2 & 0\end{array}$

C

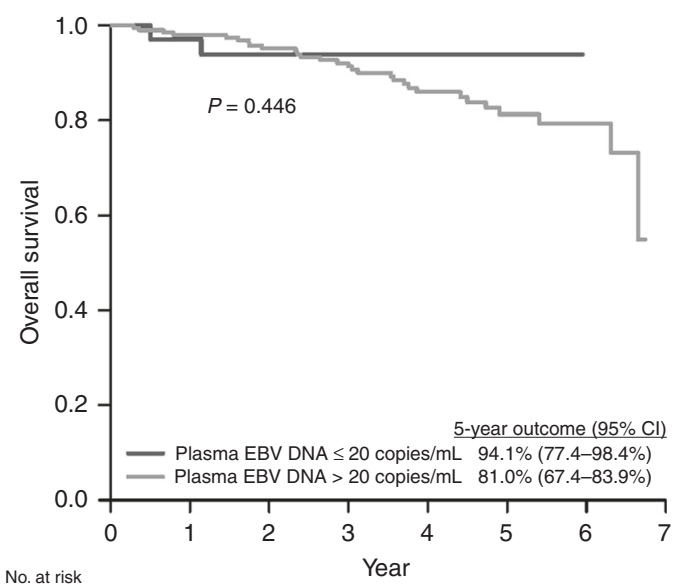

EBV DNA $\leq 20$ copies $/ \mathrm{mL}$

EBV DNA $>20$ copies $/ \mathrm{mL} 200$ b

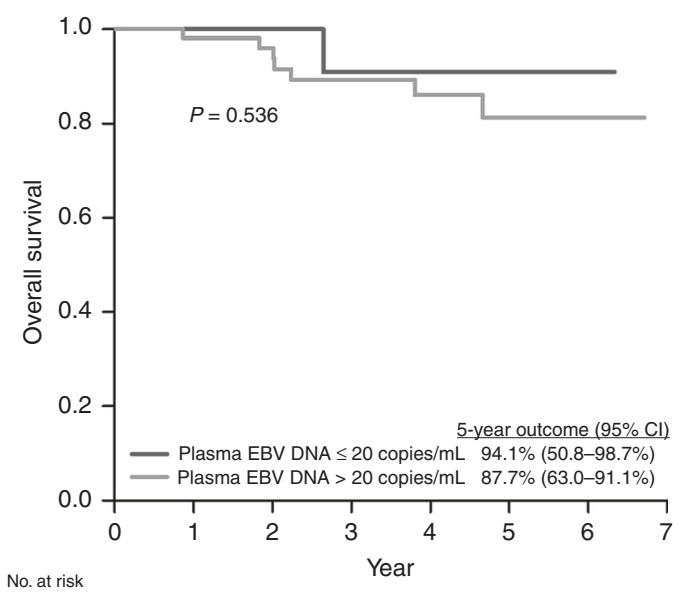

$\begin{array}{lllllllll}\text { EBV DNA } \leq 20 \text { copies } / \mathrm{mL} & 17 & 17 & 13 & 10 & 5 & 1 & 1 & 0\end{array}$

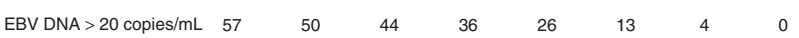

d

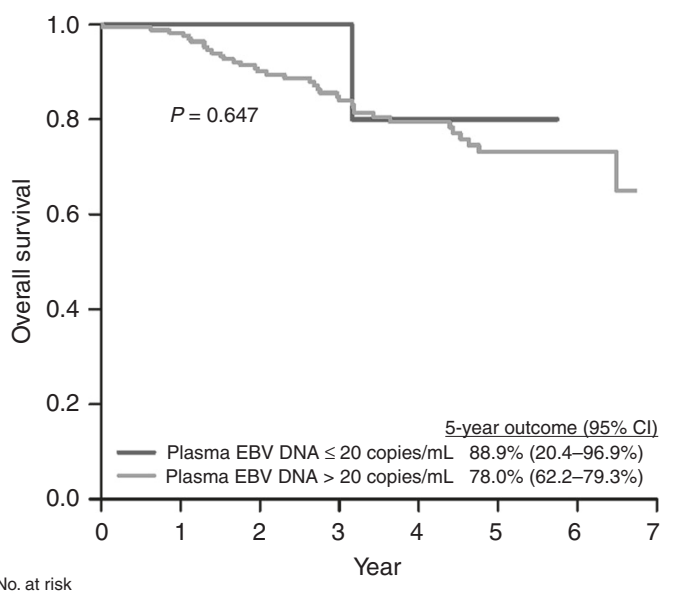

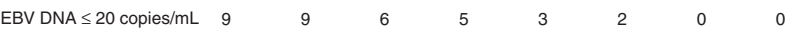

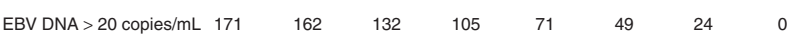

Fig. 3 Overall survival of NPC patients in the study population stratified by pre-treatment plasma EBV DNA (0-20 copies/ml vs $>20$ copies/ml). a Stage I. b Stage II. c Stage III. d Stage IVA

of median EBV DNA levels present in these patients (14-2500 copies $/ \mathrm{ml}){ }^{37}$ In a recent review of the usefulness of liquid biopsy for the detection of tumours, ${ }^{13}$ it was suggested that one of the factors affecting the effectiveness of a cancer screening test was the weight, or volume of the tumour. In patients with a tumour weight of $10 \mathrm{~g}$ and sampling $10 \mathrm{ml}$ of blood, the cancer screening test appears effective, but in patients with tumour weight less than that, the effectiveness was called into question. Since Table 1 shows that the primary tumour GTV of our EBV negative cohort was $4.4 \mathrm{~g}$ compared to $10 \mathrm{~g}$ for the EBV positive patients, it is not surprising that the no EBV can be detected in the plasma. Further data has demonstrated that when the fraction of tumour DNA drops below $0.01 \%$, then the use of $10 \mathrm{ml}$ of blood ( $4 \mathrm{ml}$ plasma) will not contain a single cancer genome. ${ }^{13,38}$

Since detection of plasma EBV may be problematic in patients with low volume early-stage disease, one would expect a better correlation in high tumour volume patients and Figure S2 indeed does show a good correlation. Our ISH results show that one possible hypothesis to explain this low or absent detectable plasma EBV DNA is because of tumour heterogeneity in EBV copy number. The studies from Chan and Lo et al. have assumed that each tumour cell contains 50 copies EBV/cell. ${ }^{12}$ However, the original publication, found a great range of EBV genome equivalents (2-137) in NPC patients. ${ }^{39}$ In addition to variable but multiple copies of the genome in each cell, there are also variable but multiple copies of the promoter $\mathrm{Wp}$ within each genome. ${ }^{40,41}$ The standard B95.8 cell line contains 11 reiterations of Bam-W, Raji cell line 7 copies and clinical isolates a mean of 6 , with a range of $5-11 .{ }^{42}$ We found in many tumours that had no detectable plasma EBV DNA there was a degree of variable EBER signal in the tumour cells -in some cases the signal was located within the nucleolus, in others the whole nucleus seemed to have positive signal, and some cells had no signal at all.

Our study demonstrated that $15.1 \%$ of histologically confirmed NPC patients were plasma EBV DNA-negative and that in these plasma EBV DNA-negative patients, $99.8 \%$ were either nonkeratinising differentiated carcinoma or undifferentiated carcinoma. In response to a review article indicating $17.2 \%-29.3 \%$ of NPC patients from endemic countries had undetectable plasma EBV at initial diagnosis, ${ }^{43,44}$ Le and colleagues suggested that some NPC may have a non-EBV origin, and the method of analysis may not be consistent. ${ }^{45}$ With respect to the possibility that NPCs may have a non-EBV origin, we found that almost all NPCs had a positive EBER ISH signal, but the signal intensity was only associated with plasma EBV DNA in those patients who had plasma EBV DNA >20 copies $/ \mathrm{ml}$. Inconsistent sample analysis is 


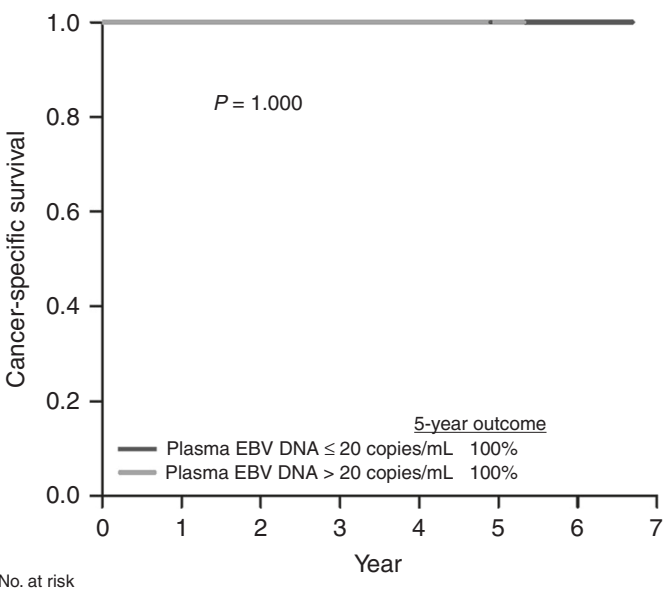

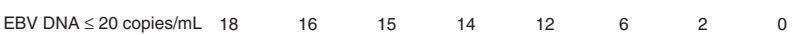

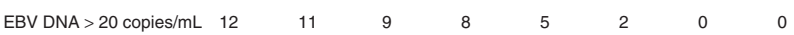

C

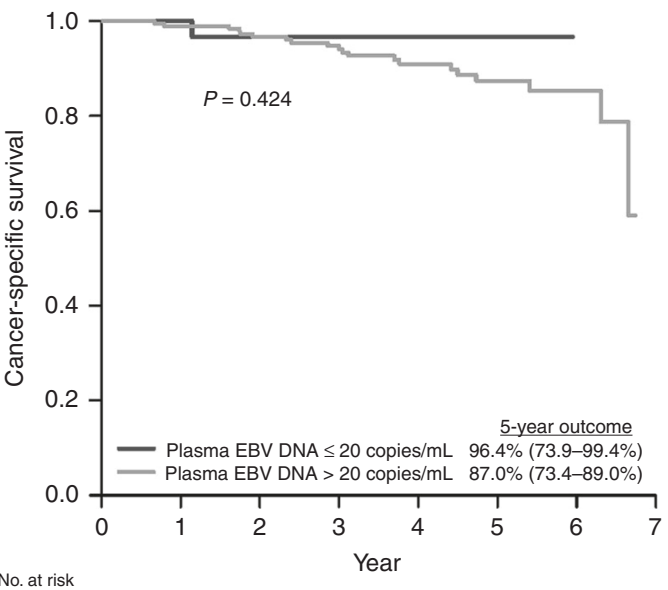

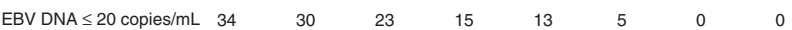

EBV DNA > 20 copies $/ \mathrm{mL} 200 \quad 188 \quad 163 \quad 138 \quad 97 \quad 55 \quad 18 \quad 18$ b

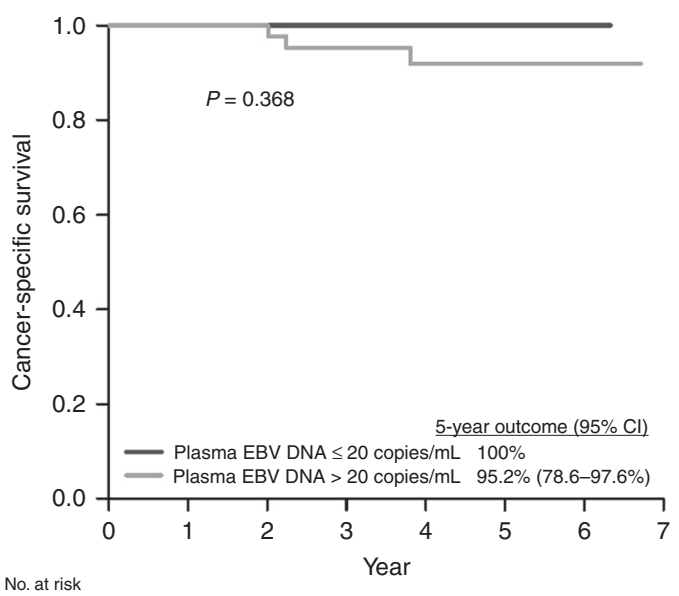

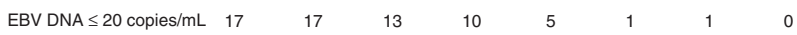

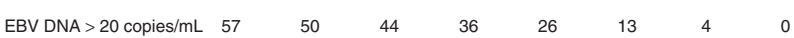

d

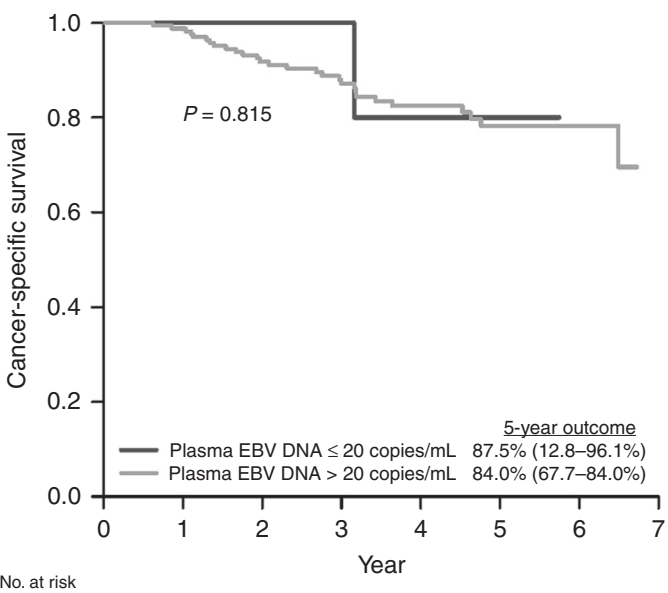

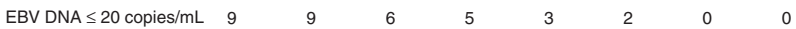

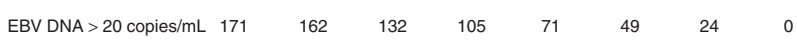

Fig. 4 Cancer-specific survival of NPC patients in the study population stratified by pre-treatment plasma EBV DNA (0-20 copies/ml vs $>20$ copies/ml). a Stage I. b Stage II. c stage III. d stage IVA

not likely as the samples in the current study were analysed by the same methodology used by Chan et al. in their screening programme. ${ }^{12}$ Technical error is also unlikely as all our cases were handled by the same laboratory with the same EBV target. All samples were analysed within 1 day of collection, so sample deterioration is unlikely to be a major factor in the evaluation of plasma EBV DNA. ${ }^{46}$ All patients who had negative plasma EBV DNA at the time of diagnosis also had negative plasma EBV DNA at subsequent testing and follow up. Similarly, all patients who had pre-treatment detectable plasma EBV DNA had its significant drop following treatment. It should be noted that even in an endemic region when the method of Lo et al. was used, 3/40 $(7.5 \%)$ cases of NPC had 0 copy $/ \mathrm{ml}$, and one of these negative cases had a positive detection when the same sample was analysed by two other laboratories. ${ }^{4}$

We demonstrated that EBER was poorly expressed in patients with low pre-treatment plasma EBV DNA (regardless of the cut-off set at 0 or 20 copies $/ \mathrm{ml}$ ). It was also poorly associated with $\mathrm{T}-\mathrm{,} \mathrm{N}-$ and overall stage of their disease even when it is advanced stage. In addition, EBER expression levels were strongly associated with pre-treatment plasma EBV DNA. Yet about half of these patients presented with advanced stage III to IVA disease at diagnosis. It can be inferred that EBV genome may be poorly incorporated in the tumour cells in this subgroup of patients leading to impaired expression of the EBER and plasma EBV DNA production. Since EBER has been demonstrated to influence the expression of RIG-I, inflammatory mediators and thus tumour progression, ${ }^{47}$ the link between EBER and tumour stage may be a reflection of the EBER induced stromal response to tumour growth leading to cell death and viral DNA release. The use of only a few slides from each patient's FFPE tumour samples for subsequent ISH for EBER which may not give an overall picture of the extent of EBV genome incorporation into every tumour cell is one study limitation, though we have already selected the most representative slides containing the greatest number of tumour cells to ensure consistent EBER scoring.

Though plasma EBV DNA has been proposed as a population screening tool for NPC by Chan et al., ${ }^{12} 62 / 518(11 \%)$ of patients had 0 copy, and 78/518 (15\%) of patients had between 0 and 20 copies/ml in our study. It appears that if this was used solely for screening in the general population, it would miss more than 130 patients with NPC each year in Hong Kong with a 7.5 million population. ${ }^{48}$ In other words, we can miss $60.0 \%, 23.0 \%, 14.5 \%$ and $5.0 \%$ of stage I, II, III and IVA NPC if only plasma EBV DNA was used as the population screening tool, running into a risk of delayed diagnosis and treatment, and the survival of these plasma 
EBV DNA-negative patients by stage was not better than those who were EBV DNA-positive, as shown in our study.

\section{CONCLUSIONS}

In conclusion, it was not uncommon for previously untreated NPC patients in endemic regions to have plasma EBV DNA below or close to the lowest limit of detection, ${ }^{49,50}$ and while plasma EBV DNA has been shown to be a reliable predictive and prognostic factor, our findings reinforced the concerns of previous authors in adopting its use for the primary diagnosis or screening of NPC in a general population. ${ }^{25,26}$ The use of EBV as a "liquid biopsy" tool thus has many of the problems associated with other cancer screening tools in terms of both sensitivity and specificity for early cancer detection and additional investigations are warranted if NPC has to be safely ruled out.

\section{ACKNOWLEDGEMENTS}

We gratefully thank all the study participants and the staff of Department of Clinical Oncology and Department of Pathology of Queen Mary Hospital of The University of Hong Kong for their contribution to this study.

\section{AUTHOR CONTRIBUTIONS}

J.M.N.: conceptualisation, investigation, data analysis, manuscript preparation and editing. V.H.F.L.: conceptualisation, investigation, data analysis, manuscript preparation and editing, review and oversight. S.K.C.: investigation, data analysis, manuscript preparation and editing. K.C.T.: investigation, data analysis, manuscript preparation and editing. C.W.C.: conceptualisation, data analysis, manuscript preparation and editing. D.L.K.: conceptualisation, data analysis, manuscript preparation and editing. K.O.L.: investigation, data analysis, manuscript preparation and editing. S.Y.C.: investigation, data analysis, manuscript preparation and editing. C.C.T.: investigation, data analysis, manuscript preparation and editing. T.H.S.: data analysis, manuscript preparation and editing, T.W.L.: investigation, data analysis, manuscript preparation and editing. M.Y.L.: investigation, data analysis, manuscript preparation and editing. P.L.K.: investigation, data analysis, manuscript preparation and editing. A.W.M.L.: investigation, data analysis, manuscript preparation and editing. All authors wrote, reviewed, critically revised and approved the final version of the manuscript.

\section{ADDITIONAL INFORMATION}

Supplementary information is available for this paper at https://doi.org/10.1038/ s41416-019-0575-6.

Competing interests: The authors declare no competing interests.

Ethics approval and consent to participate: The study protocol was approved by the Institutional Review Board of the University of Hong Kong/Hospital Authority Hong Kong West Cluster (HKU/HA HKW IRB) (IRB/REC No. UW 12-153). The study was performed in accordance with the Declaration of Helsinki. Individual written informed consent was obtained from all participating patients at enrolment.

Funding: This work was supported by Croucher Foundation and SK Yee Medical Foundation (210212, 211259 and 213208). The funder has no role in study design and conception, data collection, statistical analysis and interpretation, manuscript drafting, final manuscript approval and publication of this study.

Consent to publish: We have provided our consent to publish.

Data availability: The datasets used and/or analysed during the current study are available from the corresponding author on reasonable request.

Note: This work is published under the standard license to publish agreement. After 12 months the work will become freely available and the license terms will switch to a Creative Commons Attribution 4.0 International (CC BY 4.0).

Publisher's note Springer Nature remains neutral with regard to jurisdictional claims in published maps and institutional affiliations.

\section{REFERENCES}

1. Lee, A. W., Ma, B. B., Ng, W. T. \& Chan, A. T. Management of nasopharyngeal carcinoma: current practice and future perspective. J. Clin. Oncol. 33, 3356-3364 (2015).

2. Fung, S. Y., Lam, J. W. \& Chan, K. C. Clinical utility of circulating Epstein-Barr virus DNA analysis for the management of nasopharyngeal carcinoma. Chin. Clin. Oncol. 5, 18 (2016).

3. Kim K. Y., Le Q. T., Yom S. S., Pinsky B. A., Bratman S. V., Ng R. H. et al. Current state of PCR-based Epstein-Barr virus DNA testing for nasopharyngeal cancer. J. Natl Cancer Inst. 109, djx007 (2017).

4. Le, Q. T., Zhang, Q., Cao, H., Cheng, A. J., Pinsky, B. A., Hong, R. L. et al. An international collaboration to harmonize the quantitative plasma Epstein-Barr virus DNA assay for future biomarker-guided trials in nasopharyngeal carcinoma. Clin. Cancer Res. 19, 2208-2215 (2013).

5. Zhang, W., Chen, Y., Chen, L., Guo, R., Zhou, G., Tang, L. et al. The clinical utility of plasma Epstein-Barr virus DNA assays in nasopharyngeal carcinoma: the dawn of a new era?: a systematic review and meta-analysis of 7836 cases. Med. (Baltim.) 94, e845 (2015).

6. Zhang, J., Shu, C., Song, Y., Li, Q., Huang, J. \& Ma, X. Epstein-Barr virus DNA level as a novel prognostic factor in nasopharyngeal carcinoma: a meta-analysis. Med. (Baltim.) 95, e5130 (2016).

7. Liu, T. B., Zheng, Z. H., Pan, J., Pan, L. L. \& Chen, L. H. Prognostic role of plasma Epstein-Barr virus DNA load for nasopharyngeal carcinoma: a meta-analysis. Clin. Invest Med. 40, E1-E12 (2017).

8. Nicholls, J. M., Agathanggelou, A., Fung, K., Zeng, X. \& Niedobitek, G. The association of squamous cell carcinomas of the nasopharynx with Epstein-Barr virus shows geographical variation reminiscent of Burkitt's lymphoma. J. Pathol. 183, 164-168 (1997).

9. Pathmanathan, R., Prasad, U., Chandrika, G., Sadler, R., Flynn, K. \& Raab-Traub, N. Undifferentiated, nonkeratinizing, and squamous cell carcinoma of the nasopharynx. Variants of Epstein-Barr virus-infected neoplasia. Am. J. Pathol. 146, 1355-1367 (1995).

10. Lo, Y. M., Chan, L. Y., Chan, A. T., Leung, S. F., Lo, K. W., Zhang, J. et al. Quantitative and temporal correlation between circulating cell-free Epstein-Barr virus DNA and tumor recurrence in nasopharyngeal carcinoma. Cancer Res. 59, 5452-5455 (1999).

11. Leung, S. F., Tam, J. S., Chan, T. C., Zee, B., Chan, L. Y., Huang, D. P. et al. Improved accuracy of detection of nasopharyngeal carcinoma by combined application of circulating Epstein-Barr virus DNA and anti-Epstein-Barr viral capsid antigen IgA antibody. Clin. Chem. 50, 339-345 (2004).

12. Chan, K. C. A., Woo, J. K. S., King, A., Zee, B. C. Y., Lam, W. K. J., Chan, S. L. et al. Analysis of plasma Epstein-Barr virus DNA to screen for nasopharyngeal cancer. N. Engl. J. Med 377, 513-522 (2017).

13. Fiala, C. \& Diamandis, E. P. Circulating tumor DNA for personalized lung cancer monitoring. BMC Med 15, 157 (2017).

14. Lee, V. H., Kwong, D. L., Leung, T. W., Choi, C. W., O'Sullivan, B., Lam, K. O. et al. The addition of pretreatment plasma Epstein-Barr virus DNA into the8th edition of nasopharyngeal cancer TNM stage classification. Int J. Cancer 144, 1713-1722 (2019).

15. Lee, V. H., Kwong, D. L., Leung, T. W., Choi, C. W., Lai, V., Ng, L. et al. Prognostication of serial post-intensity-modulated radiation therapy undetectable plasma EBV DNA for nasopharyngeal carcinoma. Oncotarget 8, 5292-5308 (2017).

16. Bar-Sela, G., Kuten, A., Minkov, I., Gov-Ari, E. \& Ben-Izhak, O. Prevalence and relevance of EBV latency in nasopharyngeal carcinoma in Israel. J. Clin. Pathol. 57 290-293 (2004).

17. Henle, G. \& Henle, W. Epstein-Barr virus-specific IgA serum antibodies as an outstanding feature of nasopharyngeal carcinoma. Int J. Cancer 17, 1-7 (1976).

18. Ringborg, U., Henle, W., Henle, G., Ingimarsson, S., Klein, G., Silfversward, C. et al. Epstein-Barr virus-specific serodiagnostic tests in carcinomas of the head and neck. Cancer 52, 1237-1243 (1983).

19. Niedobitek, G., Young, L. S., Sam, C. K., Brooks, L., Prasad, U. \& Rickinson, A. B. Expression of Epstein-Barr virus genes and of lymphocyte activation molecules in undifferentiated nasopharyngeal carcinomas. Am. J. Pathol. 140, 879-887 (1992).

20. Wu, T. C., Mann, R. B., Epstein, J. I., MacMahon, E., Lee, W. A., Charache, P. et al. Abundant expression of EBER1 small nuclear RNA in nasopharyngeal carcinoma. A morphologically distinctive target for detection of Epstein-Barr virus in formalin-fixed paraffin-embedded carcinoma specimens. Am. J. Pathol. 138, 1461-1469 (1991).

21. Yeung, W. M., Zong, Y. S., Chiu, C. T., MacMahon, E., Lee, W. A., Charache, P. et al. Epstein-Barr virus carriage by nasopharyngeal carcinoma in situ. Int J. Cancer 53, 746-750 (1993).

22. Bouvard, V., Baan, R., Straif, K., Grosse, Y., Secretan, B., El Ghissassi, F. et al. A review of human carcinogens-Part B: biological agents. Lancet Oncol. 10, 321-322 (2009). 
23. Nawroz, H., Koch, W., Anker, P., Stroun, M. \& Sidransky, D. Microsatellite alterations in serum DNA of head and neck cancer patients. Nat. Med. 2, 1035-1037 (1996).

24. Chen, X. Q., Stroun, M., Magnenat, J. L., Nicod, L. P., Kurt, A. M., Lyautey, J. et al. Microsatellite alterations in plasma DNA of small cell lung cancer patients. Nat. Med. 2, 1033-1035 (1996).

25. Mutirangura, A., Pornthanakasem, W., Theamboonlers, A., Sriuranpong, V., Lertsanguansinchi, P., Yenrudi, S. et al. Epstein-Barr viral DNA in serum of patients with nasopharyngeal carcinoma. Clin. Cancer Res 4, 665-669 (1998).

26. Lo, Y. M., Chan, L. Y., Lo, K. W., Leung, S. F., Zhang, J., Chan, A. T. et al. Quantitative analysis of cell-free Epstein-Barr virus DNA in plasma of patients with nasopharyngeal carcinoma. Cancer Res. 59, 1188-1191 (1999).

27. Shao, J. Y., Li, Y. H., Gao, H. Y., Wu, Q. L., Cui, N. J., Zhang, L. et al. Comparison of plasma Epstein-Barr virus (EBV) DNA levels and serum EBV immunoglobulin A/ virus capsid antigen antibody titers in patients with nasopharyngeal carcinoma. Cancer 100, 1162-1170 (2004).

28. De Paoli, P., Pratesi, C. \& Bortolin, M. T. The Epstein Barr virus DNA levels as a tumor marker in EBV-associated cancers. J. Cancer Res. Clin. Oncol. 133, 809-815 (2007).

29. Gulley, M. L., Fan, H. \& Elmore, S. H. Validation of Roche LightCycler Epstein-Barr virus quantification reagents in a clinical laboratory setting. J. Mol. Diagn. 8, 589-597 (2006).

30. Chan, K. C. A., Chu, S. W. I. \& Lo, Y. M. D. Ambient temperature and screening for nasopharyngeal cancer. N. Engl. J. Med. 378, 962-963 (2018).

31. Wong, L. P., Lai, K. T., Tsui, E., Kwong, K. H., Tsang, R. H. \& Ma, E. S. Plasma EpsteinBarr virus (EBV) DNA: role as a screening test for nasopharyngeal carcinoma (NPC)? Int J. Cancer 117, 515-516 (2005).

32. O, T. M., Yu, G., Hu, K. \& Li, J. C. Plasma Epstein-Barr virus immunoglobulin A and DNA for nasopharyngeal carcinoma screening in the United States. Otolaryngol. Head. Neck Surg. 136, 992-997 (2007).

33. Van den Bruel, A., Jones, C., Yang, Y., Oke, J. \& Hewitson, P. People's willingness to accept overdetection in cancer screening: population survey. BMJ 350, h980 (2015).

34. Rho, J. H. \& Lampe, P. D. High-throughput analysis of plasma hybrid markers for early detection of cancers. Proteomes 2, 1-17 (2014).

35. Brodersen, J. \& Siersma, V. D. Long-term psychosocial consequences of falsepositive screening mammography. Ann. Fam. Med 11, 106-115 (2013).

36. Wu, G. X., Raz, D. J., Brown, L. \& Sun, V. Psychological burden associated with lung cancer screening: a systematic review. Clin. Lung Cancer 17, 315-324 (2016).

37. Yip, T. T., Ngan, R. K., Fong, A. H. \& Law, S. C. Application of circulating plasma/ serum EBV DNA in the clinical management of nasopharyngeal carcinoma. Oral. Oncol. 50, 527-538 (2014).
38. Abbosh, C., Birkbak, N. J., Wilson, G. A., Jamal-Hanjani, M., Constantin, T., Salari, R. et al. Phylogenetic ctDNA analysis depicts early-stage lung cancer evolution. Nature 545, 446-451 (2017).

39. Andersson-Anvret, M., Forsby, N., Klein, G. \& Henle, W. Relationship between the Epstein-Barr virus and undifferentiated nasopharyngeal carcinoma: correlated nucleic acid hybridization and histopathological examination. Int J. Cancer 20, 486-494 (1977).

40. Baer, R., Bankier, A. T., Biggin, M. D., Deininger, P. L., Farrell, P. J., Gibson, T. J. et al. DNA sequence and expression of the B95-8 Epstein-Barr virus genome. Nature 310, 207-211 (1984).

41. Allan, G. J. \& Rowe, D. T. Size and stability of the Epstein-Barr virus major internal repeat (IR-1) in Burkitt's lymphoma and lymphoblastoid cell lines. Virology 173, 489-498 (1989).

42. Sanosyan, A., Fayd'herbe de Maudave, A., Bollore, K., Zimmermann, V., Foulongne, V., Van de Perre, P. et al. The impact of targeting repetitive BamHI-W sequences on the sensitivity and precision of EBV DNA quantification. PLoS One 12, e0183856 (2017).

43. Zoto Mustafayev, T. \& Ozyar, E. In Regard to Kim et al. Int J. Radiat. Oncol. Biol. Phys. 99, 1306-1307 (2017).

44. Kim, K. Y., Le, Q. T., Yom, S. S., Ng, R. H. W., Chan, K. C. A., Bratman, S. V. et al. Clinical utility of Epstein-Barr virus DNA testing in the treatment of nasopharyngeal carcinoma patients. Int J. Radiat. Oncol. Biol. Phys. 98, 996-1001 (2017).

45. Le, Q. T., Yom, S. S., Ng, R. H. W., Bratman, S. V., Welch, J. J., Chan, K. C. A. et al. In Reply to Zoto Mustafayev and Ozyar. Int J. Radiat. Oncol. Biol. Phys. 99, 1307 (2017).

46. Ahsanuddin, A. N., Standish, M. C., Caliendo, A. M., Hill, C. E. \& Nolte, F. S. Validation of an Epstein-Barr viral load assay using the QIAGEN Artus EBV TM PCR analyte-specific reagent. Am. J. Clin. Pathol. 130, 865-869 (2008).

47. Duan, Y., Li, Z., Cheng, S., Chen, Y., Zhang, L., He, J. et al. Nasopharyngeal carcinoma progression is mediated by EBER-triggered inflammation via the RIG-I pathway. Cancer Lett. 361, 67-74 (2015).

48. Hong Kong Cancer Registry. Available at http://www3.ha.org.hk/cancereg/ default.asp. Assessed on 14 July 2018.

49. Stevens, S. J., Verkuijlen, S. A., Hariwiyanto, B., Harijadi, Paramita, D. K., Fachiroh, J. et al. Noninvasive diagnosis of nasopharyngeal carcinoma: nasopharyngeal brushings reveal high Epstein-Barr virus DNA load and carcinoma-specific viral BARF1 mRNA. Int J. Cancer 119, 608-614 (2006).

50. Tong, J. H., Tsang, R. K., Lo, K. W., Woo, J. K., Kwong, J., Chan, M. W. et al. Quantitative Epstein-Barr virus DNA analysis and detection of gene promoter hypermethylation in nasopharyngeal (NP) brushing samples from patients with NP carcinoma. Clin. Cancer Res. 8, 2612-2619 (2002). 